\title{
The bone response in endurance long distance horse
}

\author{
Mariana Damazio Rajão ${ }^{1}$, Carol S. Leite ${ }^{1}$, Kaique Nogueira ${ }^{1}$, Roberta F. Godoy ${ }^{2}$ and Eduardo Maurício Mendes \\ Lima $^{1}, *$ \\ ${ }^{1}$ Department of Veterinary Anatomy, Faculdade de Agronomia e Medicina Veterinária, Universidade de Brasília \\ (UnB), ICC Ala Sul, Campus Darcy Ribeiro, Brasilia DF 70760-701, Brazil \\ ${ }^{2}$ Writtle University College, Chelmsford CM1 3RR, United Kingdom
}

\begin{abstract}
The aim of this study was to understand the bone response against the exercise adaptations to reduce the occurrence of orthopedic injuries in endurance horses. To this end, the objective of the present work was to investigate how the bone adaptation on adult equines that were trained for $4-5 \mathrm{yr}$ to endurance races responds to the long-term exercise of moderate intensity by comparing to non-athlete horses. For this purpose, 14 Arabian horses were selected and divided equally into two groups; a control group formed by animals that had never practiced physical activity nor been tamed and an exercise group formed by athlete animals. Radiographs were obtained using a digital radiography system and penetrometer. The radiographs were stored and later processed to determine cortical bone thickness using the ProgRes ${ }^{\circledR}$ Capture Pro 2.5 (Jenoptik, Germany), cortical bone density using Adobe Photoshop CS6 (version 6.0, Adobe Systems Inc., San Jose, CA), and trabecular bone density using the Image-Pro Plus 4.1 (Media Cybernetics Inc. Silver Springs, MD). The EG presented an increase in cortical bone density and thickness compared to the control. This adaptation of the cortical bone in the exercised horses is an important factor in increasing bone resistance to exercise. These structural changes are related to a morphofunctional response by the bone tissue as a whole.
\end{abstract}

Keywords: Adaptation, Bone, Densitometry, Equine.

\section{Introduction}

Endurance riding is a relatively young sport but its popularity has been growing fast. Research into veterinary problems in endurance horses is also increasing, but there is still relatively little evidence-based information available on clinical issues, particularly orthopedic injuries. The sport has changed greatly in the past $15-20 \mathrm{yr}$ and there is now a great difference among endurance rides in different parts of the world, particularly with respect to speed (Nagy et al., 2012).

The sport has seen an increasing level of professional trainers and riders and a dramatic increase in speed at all distances, including the championship distance of $160 \mathrm{~km}$, although there has been an increase in popularity of $120 \mathrm{~km}$ single-day races. Between 1986 and 2002, winning speeds at World Equestrian Games or Endurance World Championships over a 1 d 160 $\mathrm{km}$ distance ranged from 14.8 to $17.8 \mathrm{~km} / \mathrm{h}$. However, at the 2004 World Endurance Championships held in Dubai, the average speed of the winning horse was 22.7 $\mathrm{km} / \mathrm{h}$ and $4 \mathrm{yr}$ later on the January 12, 2008, a world record of $24.7 \mathrm{~km} / \mathrm{h}$ was set; again during a race in the UAE (Marlin and Williams, 2018) and in some parts of the world, horses reach an average speed exceeding 25 $\mathrm{km} / \mathrm{h}$ over a $120-160 \mathrm{~km}$ competition distance, often galloping at more than $30 \mathrm{~km} / \mathrm{h}$ in the last phase of the competition (Nagy et al., 2012).
Lameness was the most common cause of elimination in all countries, followed by elimination for metabolic reasons $(69.2 \%$ and $23.5 \%$, respectively, of all eliminations and $31.8 \%$ and $10.8 \%$, respectively, of all started horses) (Nagy et al., 2012). Greater amounts of very high-speed running increase the volume and speed of training to get the horses fitness, consequently, the enhancement number of injuries in the endurance horses. Today, it is possible to see injuries in endurance horses that have only been seen before on racehorses (Misheff, 2010). The type and intensity of exercise and associated tissue adaptation are important factors in understanding the presumed association of exercise with orthopedic injuries (Murray et al., 2007).

Bone stress injuries are a source of concern in long-distance runners, not only because of their frequency and the morbidity they cause but also because of their tendency to recur (Marlin and Williams, 2018) and to the catastrophic consequences (Nagy et al., 2012). Optimizing bone development is based on the premise that greater bone strength should bestow greater resistance to high forces and possibly reduce orthopedic disease both in later phases of life (Helminen et al., 2000) and in horses during athletic endeavor, and so possibly mitigate musculoskeletal causes of wastage in the equine industry due to bone failure (Verheyen and Wood, 2004). 
Stress is required to maintain bone mass and to optimize load capacity during exercise. The stress causes micro-fractures that activate osteoclasts and osteoblasts, thus leading to bone remodeling (Nielsen et al., 1997; Gong et al., 2010). Bone mineral density (BMD), defined as the mineral concentration per bone volume, is altered during the bone remodeling process. The minerals are incorporated into the organic matrix of the skeleton to increase hardness and rigidity and are also directly related to bone material properties and histological characteristics (MCclure et al., 2001). Low BMD was associated with the incidence of acute fractures during an athlete's sports in humans (Oddbjørn Klomsten, 2017).

During the growth phase, the mass of the skeleton increases since the formation exceeds the resorption rate. These changes in bone tissue may also be induced by exercise (Firth, 2006); therefore, when dealing with animal athletes, understanding the adaptations of equine bone structure is important to prevent bone lesions and protect other structures of the skeletal muscle system as well.

The aim of this study was to understand the bone against the exercise adaptations to reduce the occurrence of orthopedic injuries in endurance horses. To this end, the objective of the present work was to investigate how the bone adaptation on adult equines that was trained for $4-5 \mathrm{yr}$ to endurance races responds to the long-term exercise of moderate intensity by comparing to non-athlete horses. The study evaluated cortical bone thickness and density, as well as the trabecular bone percentage in the radius, metacarpal III, accessory carpal bones, and calcaneus bones of horses that participated in endurance (120 and $160 \mathrm{~km})$ competitions in 2016 and then compared the data to those of non-trained horses.

\section{Materials and Methods}

\section{Experimental design}

The study was conducted in a stud farm where the horses were reared free on pasture. The horses were fed commercial concentrate at a $1 \%$ live weight ratio and Tifton 85 grass, while mineral salt and water were supplied ad libitum. Fourteen Arabian horses, three castrated males, and 11 females who were between 8 and $10 \mathrm{yr}$ old were evaluated. The animals were divided into two groups. The control group (CG) consisted of seven animals raised on pasture which had never been tamed nor had practiced any type of physical activity during their lives. The exercise group (EG) consisted of seven athlete horses also raised free on pasture and was undergoing a training program during at minimum $4 \mathrm{yr}$ and participating in 120 and $160 \mathrm{~km}$ endurance competition organized by the International Equestrian Federation during 2016. The program of training of the $\mathrm{CG}$ consisted of 5 days a week training and $2 \mathrm{~d}$ rest. The training was about $1 \mathrm{~h}$ and 30 min walking with 2 days a week galloping
Table 1. Radiographic technique used to capture images according to the different bones evaluated.

\begin{tabular}{lcc}
\hline \multirow{1}{*}{ Bone } & \multicolumn{2}{c}{ Technique } \\
& Dorsopalmar & Lateromedial \\
\hline Radius & - & $76 \mathrm{kv} / 4.00 \mathrm{mAs}$ \\
$\begin{array}{l}\text { Third } \\
\text { metacarpal }\end{array}$ & $74 \mathrm{kv} / 3.20 \mathrm{mAs}$ & $74 \mathrm{kv} / 2.50 \mathrm{mAs}$ \\
Calcaneus & - & $74 \mathrm{kv} / 3.20 \mathrm{mAs}$ \\
$\begin{array}{l}\text { Accessory } \\
\text { carpal }\end{array}$ & - & $76 \mathrm{kv} / 4.00 \mathrm{mAs}$ \\
\hline
\end{tabular}

about $15-30 \mathrm{~km}$. The 120 horses made one training of $60 \mathrm{~km} 4 \mathrm{wk}$ before the race and for $160 \mathrm{~km}$, the horses made on the training of $80 \mathrm{~km} 4$ wk before the race. The experimental procedures followed the guidelines of the Ethics Committee for Animal Use from the University of Brasilia, protocol number $160100 / 2013$.

Only non-lame animals took part in the experiment. A specific clinical examination of the locomotor system was performed to verify the health status of the animals, as described by Keegan et al. (2010).

\section{Radiographic evaluations}

Radiographs of the right and left thoracic and pelvic limbs were performed using a digital radiography system (Xamaru $^{\circledR}$ 1210PCB Direct Radiology, Rayence Co., Ltd.-Vatech, South Korea), with a $60-\mathrm{cm}$ focus-film distance, measured by the focus of the X-ray machine (Orange ${ }^{\circledR} 8016 \mathrm{HF}$ ). The aluminum alloy penetrometer (Varanda et al., 2016) was placed medially and dorsally to the limb to obtain the dorsopalmar and lateromedial images, respectively. The images were captured in the dorsopalmar and lateromedial projections of the third metacarpal bone and lateromedial of the radius, accessory carpal, and calcaneus bones in the projections as indicated in Table 1.

For the dorsopalmar images, the flat panel was positioned against the palmar aspect of the limb while the X-ray beam was directed parallel to the ground and perpendicular to the bone. In the lateromedial images, the plate was positioned in the medial aspect of the limb while the beam was also directed parallel to the ground and perpendicular to the bone. For comparison purposes, the bones were divided into two groups according to their morphological classification: long bones, including the radius and metacarpal III, and short bones, represented by the accessory carpal and calcaneus bone. The obtained images were stored as DICOM for further analysis. The X-ray was made after the horses complete successfully the long distance race.

\section{Quantitative evaluations of bones}

\section{Cortical bone thickness}

The images captured in the lateromedial projections were processed in the ProgRes ${ }^{\circledR}$ Capture Pro 2.5 software (Jenoptik, Germany), according to the methods described by Zarski et al. (2011). This software allowed 
standardizing a certain distance within the image: the "measure" tool in millimeters ( $\mathrm{mm}$ ) was used to standardize the penetrometer width and, consequently, the evaluated images. This procedure was necessary so that the film focus distance of approximately $60 \mathrm{~cm}$, obtained from a distance determined by the projector, would not influence data reading. From this, the cortical bone width was measured at different points (Fig. 1). The selections of the bone regions were analyzed from the lateromedial images, where the six distinct points of the diaphysis cortical of the radius and the metacarpal III, four of the calcaneus bone, and three

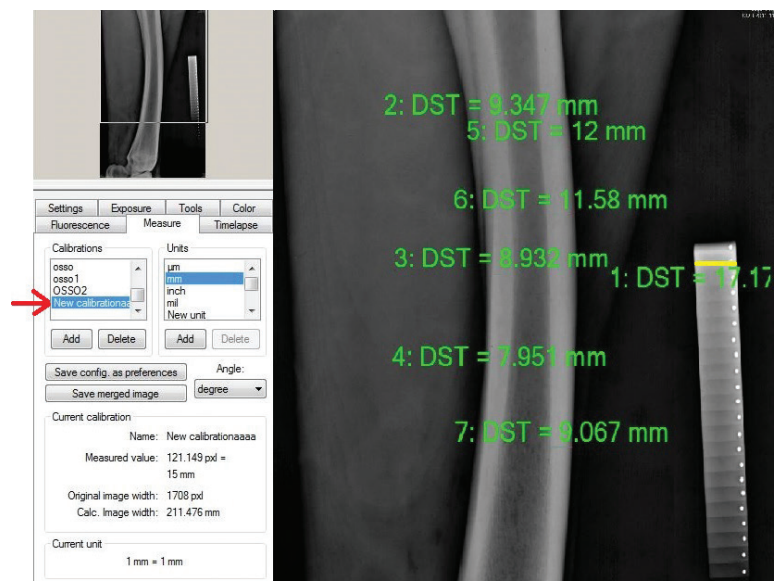

Fig. 1. Radiographic image of the radius showing how the calibration and measurement of the cortical thickness were performed at pre-determined points, using the penetrometer as a reference measurement, with images in the ProgRes ${ }^{\circledR}$ Capture Pro 2.5 software.

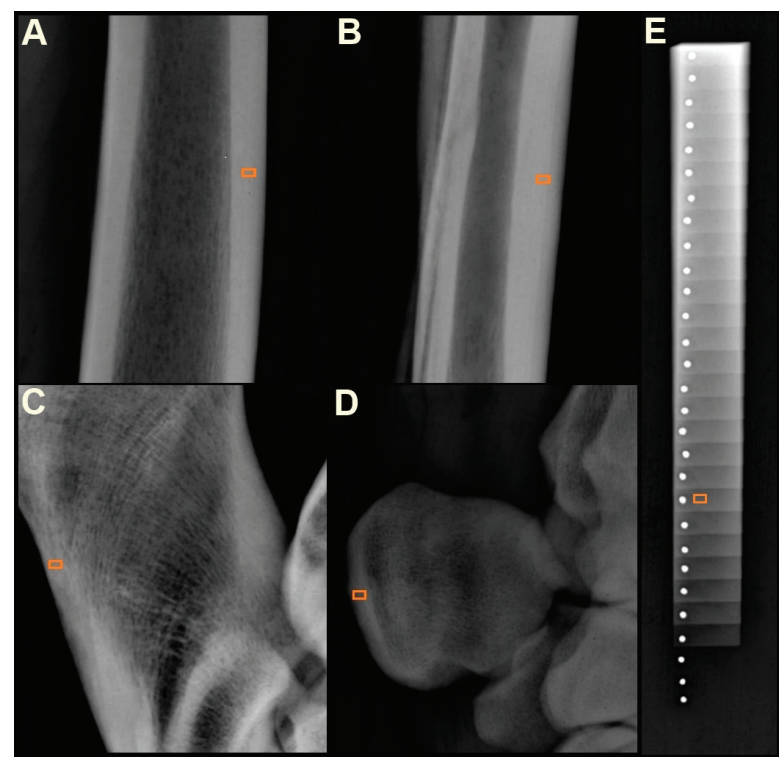

Fig. 2. Radiographic image of the radius (A), metacarpal III (B), calcaneus (C), and carpal accessory (D) bones showing the measurement point in rectangular area (orange), compared to the density at the 10th step of a penetrometer (E). points of the accessory carpal bone were included for each image. For radio and metacarpal III sound, three distinct points were used in the dorsal cortex and three non-caudal/palmar points of the bone. The reference point was the middle region of the bone and from this form marked a proximal and a distal point for analysis. For the calcaneus were marked two points in the middle part in the dorsal and palmar cortex and in the palmar part plus a proximal and distal point. For the accessory carpal bone were marked three distinct points taking the medial part of the cortex as a reference and one more proximal point and another distal point of this.

\section{Cortical bone density}

The radiographic optical densitometry was evaluated using the specific aluminum alloy penetrometer, with 25 steps, measuring $5 \times 18 \mathrm{~mm}$ (frontal area) each. The steps started at $1 \mathrm{~mm}$ thick, increasing $1 \mathrm{~mm}$ each step up to the 25th (Varanda et al., 2016). The penetrometer was positioned medially and dorsally in the dorsopalmar and lateromedial images, respectively. The captured images were processed in the Adobe Photoshop CS6 software (version 6.0, Adobe Systems Inc., San Jose, CA), according to Varanda et al. (2016). On the open image, a standard rectangle measuring $20 \times 10$ pixels was selected and dragged over an area of interest for the analysis (Fig. 2). The histogram tool was used to measure the radiographic density (mean and standard deviation) of the cortical bones at different points, which appears in different shades of gray. Subsequently, the rectangular shape was moved to the step corresponding to $10 \mathrm{~mm}$ of aluminum (10th step/degree), and the radiographic density (mean and standard deviation) corresponding to $10 \mathrm{~mm}$ of aluminum ( $\mathrm{mm} \mathrm{Al}$ ) was measured. The bone area density in $\mathrm{mm} \mathrm{Al}$ was calculated by the simple rule of three.

The diaphyseal cortical thickness was measured in six, four, and three points in the radius and metacarpal III, calcaneus and carpal accessory bones, respectively, using the lateromedial images. A descriptive analysis of the data was conducted to determine the mean and standard deviation.

\section{Trabecular bone density}

The captured images were processed in Adobe Photoshop CS6 (version 6.0, Adobe Systems Inc., San Jose, CA), according to Mahl et al. (2006). Cross-sectional scan slices, representing the trabecular tissue of the long and short bones, were obtained from the lateromedial images of the radius, accessory carpal and calcaneus bones, and the dorsopalmar images of metacarpal III. Three cross-sections were performed for each image (Fig. 3) and processed in the Image-Pro Plus 4.1 software (Media Cybernetics Inc., Silver Springs, MD) according to Blatt et al. (2004). Each cross-section was processed in Image-Pro Plus 4.1 using the "Perform Segmentation" tool to determine the mean and the standard deviation of the evaluated segment. 


\section{Statistical analysis}

Descriptive analysis was applied to obtain the mean and standard deviation. Normality was assessed using the Kolmogorov-Smirnov test. The data were non-parametric and were evaluated by the Mann-Whitney test. The analysis was performed using Sigma-Stat 3.5 software at $p \leq 0.05$.

\section{Results}

\section{Evaluation of the cortical thickness}

The cortical thickness of the radius and metacarpal III increased $(p \leq 0.05)$ significantly compared to control. However, the short bones were not significantly different between the horses of the exercise and CGs (Table 2).

\section{Evaluation of the cortical and trabecular bone density}

The densitometry of the long bones showed that the cortical bone density of metacarpal III increased significantly $(p \leq 0.05)$ in the exercised animals compared to the $C G$. The radius was not significantly different between the two groups. For the short bones, the cortical bone density in the calcaneus and accessory carpal increased $(p \leq 0.05)$ in the horses of the EG compared to the control (Table 3).

The mean trabecular percentages (\%) of the long and short bones were not significantly different between groups (Table 4).

\section{Discussion}

Differences have been seen in bone mass and bone turnover between human power athletes (sprinters, jumpers, hurdlers, and multi-event athletes), endurance athletes (middle distance runners and distance runners), and nonathlete controls, suggesting that different strain types and magnitude cause bone remodel at different rates. Equine athletes also train for different sports types

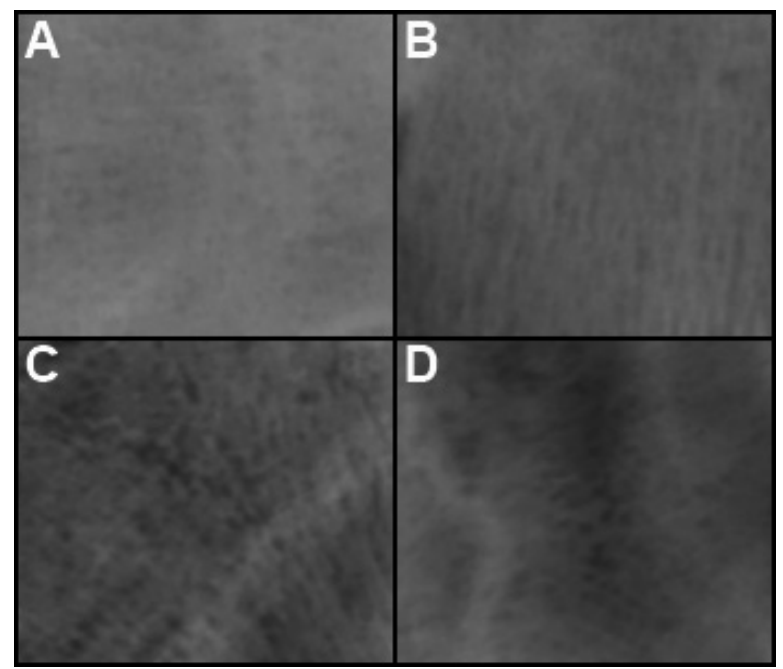

Fig. 3. Cross-sections of the trabecular bone of the radius (A), metacarpal III (B), calcaneus (C), and carpal accessory (D) obtained using Adobe Photoshop CS6. and intensities, but there has been a little investigation on the effect of training for different sports and levels predisposed to damage (Murray et al., 2007).

With the increase of the speed in races, endurance horses under certain training and race conditions are exposed to the same high levels of distal limb loading cycles found in thoroughbred racehorses (Bolwell et al., 2012). Racing at faster speeds over variable terrain causing an increased concussion in the feet which could also contribute to the high incidence of gait-related eliminations reported. The magnitude of loading in the distal limb is influenced by the speed of the gait selected, as well as ground surface and the

Table 2. Means and standard deviations of the cortical thickness in millimeters $(\mathrm{mm})$ of the radius, third metacarpal, calcaneus, and accessory carpal bone.

\begin{tabular}{lcc}
\hline \multicolumn{3}{c}{ Cortical thickness } \\
\hline \multicolumn{1}{c}{ Bone } & GC & EG \\
Radius & $6.46 \pm 1.40^{\mathrm{a}}$ & $6.97 \pm 1.20^{\mathrm{b}}$ \\
\hline Third metacarpal & $4.49 \pm 2.38^{\mathrm{a}}$ & $5.76 \pm 2.38^{\mathrm{b}}$ \\
Calcaneus & $3.41 \pm 1.24^{\mathrm{a}}$ & $3.85 \pm 1.4^{\mathrm{a}}$ \\
\hline Accessory carpal & $4.0 \pm 1.11^{\mathrm{a}}$ & $3.6 \pm 0.90^{\mathrm{a}}$ \\
\hline
\end{tabular}

Different letters between the columns indicate significant differences $(p \leq 0.05)$. GC, control group; EG, exercise group.

Table 3. Means and standard deviations of the cortical density in millimeters of aluminum $(\mathrm{mmAl})$ of the radius, third metacarpal, calcaneus, and accessory carpal bone.

\begin{tabular}{lcc}
\hline \multicolumn{3}{c}{ Cortical density } \\
\hline \multicolumn{1}{c}{ Bone } & GC & EG \\
\hline Radius & $300 \pm 64.36^{\mathrm{a}}$ & $327.2 \pm 108.5^{\mathrm{a}}$ \\
\hline Third metacarpal & $157.6 \pm 21.69^{\mathrm{a}}$ & $186.4 \pm 27.41^{\mathrm{b}}$ \\
\hline Calcaneus & $151.6 \pm 28.19^{\mathrm{a}}$ & $203.5 \pm 84.56^{\mathrm{b}}$ \\
\hline Accessory carpal & $149.4 \pm 25.26^{\mathrm{a}}$ & $183.5 \pm 55.71^{\mathrm{b}}$ \\
\hline
\end{tabular}

Different letters between the columns indicate significant differences $(p \leq 0.05)$. GC, control group; EG, exercise group.

Table 4. Means and standard deviations of the trabecular bone percentage (\%) of the radius, third metacarpal, calcaneus, and accessory carpal bone.

\begin{tabular}{lcc}
\hline \multicolumn{3}{c}{ Trabecular bone percentage } \\
\hline \multicolumn{1}{c}{ Bone } & GC & EG \\
Radius & $54.54 \pm 10.66^{\mathrm{a}}$ & $51.21 \pm 11.28^{\mathrm{a}}$ \\
$\begin{array}{l}\text { Third } \\
\text { metacarpal }\end{array}$ & $45.4 \pm 13.34^{\mathrm{a}}$ & $45.50 \pm 13.38^{\mathrm{a}}$ \\
Calcaneus & $53.95 \pm 14.7^{\mathrm{a}}$ & $54.91 \pm 11.37^{\mathrm{a}}$ \\
$\begin{array}{l}\text { Accessory } \\
\text { carpal }\end{array}$ & $45.63 \pm 9.36^{\mathrm{a}}$ & $49.90 \pm 8.77^{\mathrm{a}}$ \\
\hline
\end{tabular}

Different letters between the columns indicate significant differences $(p \leq 0.05)$. GC, control group; EG, exercise group. 
accumulative effects of repetitive training and racing (Marlin and Williams, 2018).

Greater amounts of very high-speed running may be associated with increased injury risk; however, there is evidence of lower injury risk when players performed greater amounts of low-intensity activity and short acceleration efforts (Gabbett, 2016). Different exercise intensities and types may induce particular site-specific strains, influencing osteochondral adaptation and potentially predisposing to injury (Murray et al., 2007). In the quest for reducing musculoskeletal injuries in the face of the training, many studies evaluated the bone and their adaptations. These studies documented the response to short-duration exercise of up to 6 months of training (Boyde, 2003; Hiller et al., 2003; Firth, 2006; Firth et al., 2011). However, to our knowledge, there are no studies comparing the effects of long-term exercise on the bones of horses undergoing long distance training $(120$ and $160 \mathrm{~km})$. Thus, the ways the bone responds to exercise were evaluated in endurance horses conditioned and subjected to long-distance and moderate intensity exercise. The results of horses which have worked for 4-5 yr in this condition were compared to those of horses not submitted to any training program to determine and understand the modifications that exercise could promote in different bones.

For this, the bones were chosen by considering the positioning, morphology, and ossification type (endochondral or epiphyseal) in addition to their accessibility for obtaining the images (Skedros et al., 2009; Firth et al., 2011). Skedros et al. (2009) stated that the radius represents long curved bones that are subject to torsion or flexion, whereas metacarpal III represents long straight bones that twist, while the calcaneus is a short bone subject to flexion. Other authors have used the accessory carpal bone to standardize efficiently the measurement of BMD in athlete horses because it is an easily accessible bone (Vulcano and Santos, 2003). In the present work, the radius and metacarpal III represent the long bones, while the accessory carpal and calcaneus bones represent the short ones.

In general, physical exercise promotes bone adaptations (Hiller et al., 2003; Firth, 2006; Gong et al., 2010; Firth et al., 2011). The cortical thickness of the long bones of endurance horses increased $7.89 \%(p \leq 0.05)$ in the radius and $28.28 \%$ in metacarpal III compared to the non-athletes of the CG. Similarly, Firth et al. (2011; 2012) reported greater cortical bone thickness in the metacarpal III of English thoroughbred horses submitted to exercise. It is believed that the bone remodeling characteristics were controlled primarily by micro-lesions caused in regions subject to intense stresses due to exercise (Gong et al., 2010).

Functionally and morphologically, considering the long bones used in this study, the greater cortical bone thickness observed in animals submitted to exercise contributed to increasing the resistance to flexion and torsion as proposed by Firth (2006). This greater cortical thickness increased the cortical area in the transversal axis, causing an increase in mineral bone density and bone resistance to the pressure as well. Therefore, we believe that the increased cortical bone quantity/density and quality conferred greater resistance to athlete horses compared to the animals of the CG by increasing the bone resistance to the stress arising from imposed exercise.

The cortical bone density increased $18.27 \%(p \leq 0.05)$ in metacarpal III, $34.23 \%$ in the calcaneus, and $22.82 \%$ in the carpal accessory $(p \leq 0.05)$ of the athlete horses (EG) compared to the controls. Gong et al. (2010) suggested that, in general, this process of increasing bone density in certain bones and bone regions occurs throughout the entire life of the animal and is an important way of renewing and maintaining bone mass. Hiller et al. (2003) evaluated the metacarpal III of exercised animals and reported that exercise was able to inhibit osteoclastic bone absorption, thus establishing a compensatory mechanism of the organism to deposit more and lose less bone mass, leading to increased local mineral bone density. This increase in bone density was important to increase bone strength and decrease bone susceptibility to injury.

In humans, studies showed a significant increase in the femoral BMD (whether measured by $\mathrm{g} / \mathrm{cm}^{2}$ or $Z$-score) in runners compared to nonrunners. This can be explained by the fact that biomechanically, femoral was subjected to compressive and shear forces during running. These forces produce higher strain level and hence a greater osteogenic stimulus (Shafshak et al., 2017). Other study find that endurance athletes had higher BMD than controls in lower limb sites only when power athletes had higher regional BMD at lower limb, lumbar spine, and upper limb sites (Bennell et al., 1997). Number of training hours and acute fractures were significantly associated with low BMD. However, both factors become insignificant when controlling for the type of sport (Oddbjørn Klomsten, 2017). The trabecular bone percentage was not significantly different between the horses of both groups. Likewise, other studies comparing the metacarpal III, proximal phalanx, and carpal III of thoroughbred horses, with and without forced exercise, reported no increased trabecular bone tissue as a response or adaptation to exercise (Firth et al. 2011; 2012). Firth et al. (2011) evaluated racehorses reared on pasture, with and without forced exercise, and observed smaller trabecular bone in exercised horses along with a larger cortical bone area. From these findings, Firth et al. (2011) concluded that the external part of the bone of athlete horses was more rigid and protected the center of the bone, resulting in less pressure and, therefore, a reduced response to the tissue mineralization of the central part of the bone. 
In contrast, Boyde (2003) evaluated the metacarpal III of English thoroughbred horses, exercised and not exercised, and reported that the exercise significantly increased the trabecular bone, which acted as a compensatory mechanism in the exercised group compared to the control. In the present study, the similar trabecular bone observed between the two groups can be understood as proposed by Firth et al. (2011): the trabeculae acted as a deposit for the bone matrix, guaranteeing an increase in the cortical thickness, with no difference between them.

In general, to our knowledge, the literature search on studies regarding bone adaptations as a response to exercise showed a lack of research using adult animals between 8 and 10 yr old. Moreover, the bone response to exercise corroborated the results reported for young animals (Boyde, 2003; Hiller et al., 2003; Firth et al., 2011). Bogers et al. (2014) suggested that the effect of long-term exercise did not promote a significant change compared to the changes observed at the beginning of the exercise, thus suggesting that these modifications occurred as a result of physical exercise, regardless of animal age.

\section{Conclusion}

The results showed that the changes observed between the exercised group and the CG resulted from a long-term exercise training of moderate intensity. The most significant change was observed for the cortical bone thickness of long bones and density of third metacarpal and short bones; the cortical bone thickness and density increased in exercised animals compared to the control. On the other hand, the trabecular bone was similar between the groups. It is believed that the cortical bone may have shielded the trabecular bone, thus restraining its movement and load, limiting its adaptation. However, further studies are recommended to elucidate which factors led to these results. It is concluded that physical effort changed the quantity and quality of the cortical bones of athlete horses that competed in 120-160 km endurance races, without, however, modifying the structure of the trabecular bone. These changes are related to a morphofunctional response of the bone tissue as a whole.

\section{References}

Bennell, K.L., Malcolm, S.A., Khan, K.M., Thomas, S.A., Reid, S.J., Brukner, P.D., Ebeling, P.R. and Wark, J.D. 1997. Bone mass and bone turnover in power athletes, endurance athletes, and controls: a 12-month longitudinal study. Bone 20, 477-484.

Blatt, R.J., Clark, A.N., Courtney, J., Tully, C. and Tucker, A.L. 2004. Automated quantitative analysis of angiogenesis in the rat model using Image-Pro Plus 4.1. Comput. Methods Programs Biomed. 75, 75-79.

Bogers, S.H., Rogers, C.W., Bolwell, C.F., Roe, W.D., Gee, E.K. and McIlwraith, C.W. 2014. Impact of race training on volumetric bone mineral density and its spatial distribution in the distal epiphysis of the third metatarsal bone of 2-year-old horses. Vet. J. 201, 353-358.

Bolwell, C.F., Rogers, C.W., French, N. and Firth, E.C. 2012. Associations between yearling exercise and interruptions to race training in thoroughbred racehorses. Am. J. Vet. Res. 73, 1610-1616.

Boyde, A. 2003. The real response of bone to exercise. J. Anat. 203, 173-189.

Firth, E.C. 2006. The response of bone, articular cartilage and tendon to exercise in the horse. J. Anat. 208, 513-526.

Firth, E.C., Rogers, C.W., Van Weeren, P.R., Barneveld, A., Mcilwraith, C.W., Kawcak, C.E., Goodship, A.E. and Smith, R.K. 2011. Mild exercise early in life produces changes in bone size and strength but not density in proximal phalangeal, third metacarpal and third carpal bones of foals. Vet. J. 190, 383-389.

Firth, E.C., Rogers, C.W., Van Weeren, P.R., Barneveld, A., Mcilwraith, C.W., Kawcak, C.E., Goodship, A.E. and Smith, R.K. 2012. The effect of previous conditioning exercise on diaphyseal and metaphyseal bone to imposition and withdrawal of training in young Thoroughbred horses. Vet. J. 192, 34-40.

Gabbett, T.J. 2016. The training - injury prevention paradox: should athletes be training smarter and harder? Br. J. Sports Med. 50, 273-280.

Gong, H., Zhu, D., Gao, J.L.V.L. and Zhang, X. 2010. An adaptation model for trabecular bone at different mechanical levels. BioMed. Eng. [Online] 9, 32. https://doi.org/10.1186/1475-925X-9-32

Helminen, H.J., Hyttinen, M.M., Lammi, M.J., Arokoski, J.P., Lapvetelainen, T., Jurvelin, J., Kiviranta, I. and Tammi, M.I. 2000. Regular joint loading in youth assists in the establishing and strengthening of the collagen network of articular cartilage and contributes to the prevention of osteoarthrosis later in life: a hypothesis. J. Bone Miner. Metab. 18, 245-257.

Hiller, L.P., Stover, S.M., Gibson, V.A., Gibeling, J.C., Prater, C.S., Hazelwood, S.J., Yeh, O.C. and Martin, R.B. 2003. Osteon pullout in the equine third metacarpal bone: Effects of ex vivo fatigue. J. Orthop. Res. 21, 481-488.

Keegan, K.G., Dent, E.V., Wilson, D.A., Janicek, J., Kramer, J., Lacarrubba, A., Walsh, D.M., Cassells, M.W., Esther, T.M., Schiltz, P., Frees, K.E., Wilhite, C.L., Clark, J.M., Pollitt, C.C., Shaw, R. and Norris, T. 2010. Repeatability of subjective evaluation of lameness in horses. Equine Vet. J. 42, 92-97.

Mahl, C.R.W., Fountoura, F.G., Borelli, P.V., Silva, I.L. and Fontanella, V. 2006. Mandible's radiographic and histomorphometric assessment in female rats medicated with glucocorticoid and biphosphonate. Rev. Fac. Odontol. 50, 16-19. 
Marlin, D. and Williams, J. 2018. Equine endurance race pacing strategy differs between finishers and non-finishers in $120 \mathrm{~km}$ single-day races. Comp. Exerc. Physiol. 14, 11-18.

McClure, R.S., Glickman, L.T., Glickman, N.W. and Weaver, C.M. 2001. Evaluation of dual energy X ray absorptiometry for in situ measurement of bone mineral density of equine metacarpi. Am. J. Vet. Res. 62, 752-756.

Misheff, M.M. 2010. Lameness in endurance horses. In: Diagnosis and management of lameness in the horse, 2nd Ed. St. Louis, MO: Elsevier Saunders, pp: 1137-1148.

Murray, R.C., Branch, M.V., Dyson, S.J., Parkin, T.D.H. and Goodship, A.E. 2007. How does exercise intensity and type affect equine distal tarsal subchondral bone thickness? J. Appl. Physiol. 102, 2194-2200.

Nagy, A., Dyson, S.J. and Murray, J.K. 2012. Veterinary review of endurance riding as an international competitive sport. Vet. J. 194, 288-293.

Nielsen, B.D., Potter, G.D., Morris, E.L., Odom, T.W., Senor, D.M., Reynolds, J.A., Smith, W.B. and Martin, M.T. 1997. Changes in the third metacarpal bone and frequency of bone injuries in young quarter horses during race training-observations and theoretical considerations. J. Equine Vet. Sci. 17, 541-549.

Oddbjørn Klomsten, A. 2017. Bone health in Norwegian endurance athletes. Masteroppgaver/ Masters' theses, European Master in Health and
Physical Activity. Available via http://hdl.handle. net/11250/2450595

Shafshak, T.S., Rezk, M.M., El-Tawab, S.S. and Mohareb, M.M. 2017. The effect of running on femoral bone mineral density. Saudi J. Sports Med. 17, 27-31.

Skedros, J.G., Mendenhall, A.D., Kiser, C.J. and Winet, H. 2009. Interpreting cortical bone adaptation and load history by quantifying osteon morphotypes in circularly polarized light images. Bone 44, 392-403.

Varanda, L.F.O., Moraes, J.M., Mota, A.L.A.A., Barreto-Viana, A.R.C., Nogueira, K., Godoy, R.F. and Lima, E.M.M. 2006. Osteogenic effect of percutaneous implantation of mononuclear cell fraction in horse with experimental bone fracture. Asian J. Anim. Vet. Adv. 11, 198-203.

Verheyen, K.L. and Wood, J.L. 2004. Descriptive epidemiology of fractures occurring in british thoroughbred racehorses in training. Equine Vet. J. 36, 167-173.

Vulcano, L.C. and Santos, F.A.M. 2003. Determination and padronization of normal values of bone mineral density (BMD) of the acessory carpus bone in young Thorougnbred horse using optical densitometry in radiographic image. Braz. J. Vet. Res. Anim. Sci. 40, 54-61.

Zarski, D., Targonska, K., Krejszeff, S.K., Kwiatkowski, M., Kupren, K. and Kucharczyk, D. 2011. Influence of stocking density and type of feedon the rearing of crucian carp, Carassius carassius (L.), larvae under controlled conditions. Aquacult. Int. 19, 1105-1117. 